\title{
INTRACELLULAR MONITORING OF AS1411 APTAMER BY TIME-RESOLVED MICROSPECTROFLUORIMETRY AND FLUORESCENCE IMAGING
}

Authors (alphabetical order): Jiř́ Bok ${ }^{\mathrm{a}}$, Stéphanie Bonneau ${ }^{\mathrm{b}}$, Eva Kočišováa ${ }^{\mathrm{a}}$, Petr Praus ${ }^{\mathrm{a}}$, Franck Sureau $^{\mathrm{b}}$

${ }^{a}$ Charles University in Prague, Faculty of Mathematics and Physics, Institute of Physics, Prague 2, Czech Republic

${ }^{b}$ Université Pierre et Marie Curie, Laboratoire Jean Perrin, case courrier 114, 4 Place Jussieu, 75005 Paris, France

The e-mail address, telephone and fax numbers of the corresponding author

\begin{abstract}
Time-resolved micro-spectrofluorimetry and fluorescence micro imaging - two complementary fluorescence techniques - provide important information about the intracellular distribution, level of uptake and binding/interactions inside living cell of the labeled molecule of interest. They were both employed to monitor "the fate" of the aptamer AS1411 labeled by ATTO 425 in two cell lines: U87 MG tumoral cells and Hs68 non-tumoral cells. Confocal microspectrofluorometer adapted for time-resolved intracellular fluorescence measurements by using a phase-modulation principle with homodyne data acquisition was employed to acquire intracellular emission spectra. Acquired data from both the solution and the intracellular space enable to monitor the aptamer localization and its interactions with biological structures inside the cell.
\end{abstract}

Keywords: aptamer, AS1411 aptamer, intracellular fluorescence, micro imaging, phase modulation fluorescence lifetime 


\section{Introduction}

Different oligonucleotide strategies (e.g. antigen, antisense and aptamer strategy) that employ the sequences of synthesized deoxyribo- or ribonucleotides to inhibit and/or regulate cellular gene expression have been extensively studied in recent decades. ${ }^{1}$ As they foresee considerable therapeutic promise in the treatment of various hereditary and non-hereditary diseases, with the emphasis on strain of different types of the cancer recently, the unflagging interest in their research is leading. Aptamer strategy emerged at the end of the 1980s and aptamers as such became a group of potential therapeutics because they possess several biophysical and biological properties making them very attractive in pharmacological application. Aptamers (coming from the latin word "aptus" that means fit) are generally small oligonucleotides (comprising of 12-30 bases usually) or peptides that form at appropriate conditions a specific three-dimensional structure. After its binding to the key targeted protein often with a high affinity and following cellular uptake, demanded biological response in the cellular environment is elicited. ${ }^{2,3}$

The molecule of AS1411 is considered as an advanced therapeutic aptamer with potential in the anticancer treatment. ${ }^{4}$ It has been previously found that AS1411 is able to inhibit the proliferation of the cells and to induce the dead in many types of cancer cell lines. Moreover, it was demonstrated that its effect on normal cells is only little. ${ }^{2,4}$ It is a case of guanine-rich 26-mer sequence (5'-GGTGGTGGTGGTTGTGGTGGTGGTGG) with a nonmodified, i.e. natural (phosphodiester), backbone. AS1411 molecules in the presence of $\mathrm{K}^{+}$ adopt a stable dimeric quadruplex structure with surprising characteristic - the resistance to cellular and serum nucleases. This stability enables effective interaction with its primary target - nucleolin, a protein involved in different cellular processes that is present on the surface of many cells as well as in the cellular environment. ${ }^{4,5}$ Formed aptamer/nucleolin complex is suitable to cross the membrane, distributes in the intracellular environment and activates the processes leading to cell death. Mode of the penetration inside the cell is not yet completely elucidated, however the different pathways were observed for different types of the cells. Uptake of AS1411 in DU145 prostate cancer cells occurred by macropinocytosis, however in case of Hs27 nonmalignant human foreskin fibroblasts non macropinocytosis pathway was noticed. ${ }^{5}$

Advanced microfluorescence techniques provide the possibilities to monitor the distribution of molecules or structures of interest and to follow their "fate" (findings about target, its environment and related interactions) via convenient fluorescent label. Classic or confocal fluorescence micro imaging, a standard way for intracellular distribution studies, 
when completed with time-resolved microspectrofluorimetry represent a strong tool to fulfill this task. ${ }^{6}$ In this paper we introduce both mentioned techniques focused on AS1411 aptamer based synthetic molecule labeled by ATTO 425 inside the U-87 MG human brain tumor cell line. We show the aptamer distribution in the cellular environment and discuss its interaction in connection with measured fluorescence lifetimes.

\section{Materials and methods}

Synthetic 29-mer oligonucleotide with sequence 5'd(TTTGGTGGTGGTGGTTGTGGTGGTGGTGG) based on a 26-mer AS1411 (5'GGTGGTGGTGGTTGTGGTGGTGGTGG) with three thymine bases added at the 5 'end and labeled by ATTO 425 was synthesized and purified (HPLC) by Biomers, Germany. Thymine bases were inserted as a spacer to avoid fluorophore quenching. Moreover, to insure the stability of the structure, the first four linkages (from 5 end) were modified: the phosphodiester linkage was replaced by phosphorothioate. Oligonucleotide was dissolved in PBS buffer with $\mathrm{K}^{+}$at concentration $150 \mathrm{mM}$ to induce the formation of a quadruplex structure. ${ }^{7}$ Final concentration of aptamer quadruplex solution was $0.25 \mathrm{mM}$.

Tumoral cell line U-87 MG (human glioblastoma cells) and non-tumoral cell line Hs68 (human foreskin cells) were used in the experiment. Cells were cultured by standard method in $25 \mathrm{~cm}^{2}$ flasks at temperature $37{ }^{\circ} \mathrm{C}$ in a humidified $5 \% \mathrm{CO}_{2}$ atmosphere. Used Dulbecco's Modified Eagle's Medium (DMEM) supplemented with 10\% calf fetal serum, 2 $\mathrm{mM} \mathrm{L-glutamine}$, streptomycin $(0.1 \mathrm{mg} / \mathrm{ml})$ and penicillin $(100 \mathrm{U} / \mathrm{ml})$ were purchased from Sigma-Aldrich and Biomedia, France. Cells were subcultured in Petri dishes 48 hours before an experiment. Incubation with aptamer solution for about 2-3 hours before the measurement was found as sufficient. The aptamer concentration in Petri dishes was about $2 \mu \mathrm{M}$.

Our microspectrofluorometer has been built on a phase modulation principle by using a homodyne method. ${ }^{6,8}$ Its block diagram can be seen in Figure 1. The fluorescence lifetime can be determined for all emission wavelengths by acquiring several spectral images (regularly 6) by changing the phase shift of modulated excitation laser beam (master synthesizer) in relation to fixed detector gain modulation phase shift (slave synthesizer). ${ }^{9}$ To resolve multiple lifetime components of the fluorescence, the spectra acquisition was performed for each of usually 8 individual modulation frequencies covering evenly 10 to $180 \mathrm{MHz}$ interval. Analog modulated Laser Diode Module (Omicron LDM 442.50.A350) with $50 \mathrm{~mW}$ peak output (attenuated to 1 to tens $\mu \mathrm{W}$ at the sample level) was used for the 
excitation at $442 \mathrm{~nm}$ wavelength. It is driven by the master synthesizer (IFR model 2025). This excitation wavelength matches free ATTO 425 label excitation band (436 $\mathrm{nm}$ at maximum value) quite well.

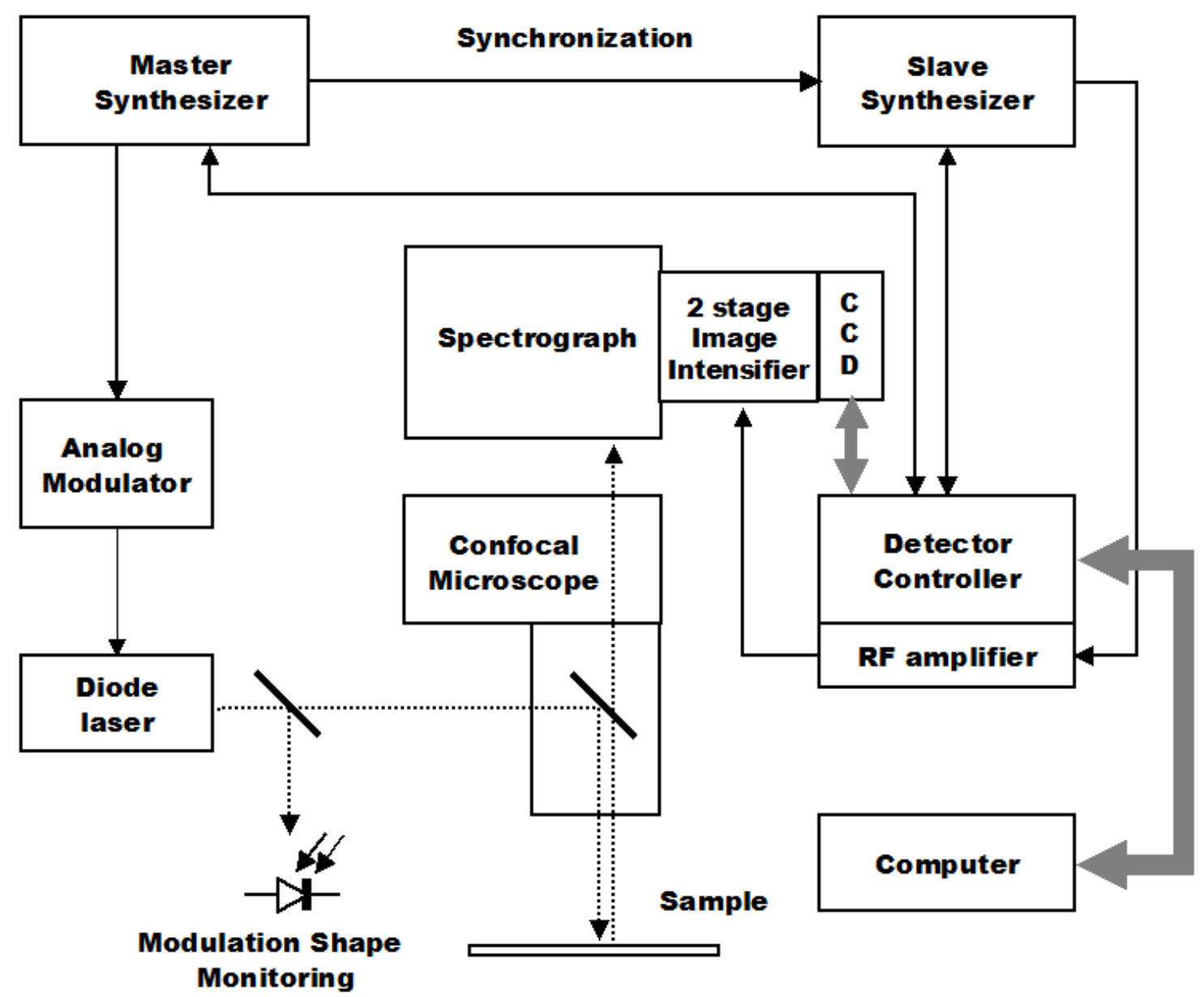

Figure 1 Block diagram of the fluorescence microspectrometer with time resolution

Confocal epifluorescence upright microscope (Zeiss UMSP-80) is used with a highnumerical-aperture water-immersion $63 \times$ objective (Zeiss Neofluar, NA 1.2). Fluorescence signal collected from the microscope pinhole is focused on the entrance slit of the Jobin-Yvon HR640 spectrograph equipped with a 100 line/mm grating. Spectral detection window (375 nm wide) is covering both the excitation wavelength (elastic scattering, used as a lifetime reference) and the fluorescence emission of the fluorophore (see Figure 2). The detection part consists of a gain modulated image intensifier driven by amplified output of the slave synthesizer (IFR, model 2025) working at precise synchronism with the master one. The 
image intensifier is optically coupled with the CCD detector $(1024 \times 1024$ pixels). The data transfer from CCD detector readout and control unit to the data acquisition computer uses USB interface. Both high frequency digital synthesizers (modulation frequency, the phase and the output level) are directly controlled by the computer via RS232C serial interface.

The spectrometer setup control and data acquisition were done by the LIFLIM software (Lambert Instruments). To calculate the phase shift and demodulation spectral dependence of the fluorescence signal from the acquired data software has been written an inhouse program PHR in our laboratory (at the Institute of Physics). For each frequency, the output data of LIFLIM are values of intensity, phase and modulation for 512 or 1024 equidistant wavelength points. In our program, the elastic scattering peak (taken as a reference) is fitted by asymmetric Lorentzian curve to find exact position of the maximum. Then, reference values of phase $\left(\mathrm{P}_{\mathrm{ref}}\right)$ and modulation $\left(\mathrm{M}_{\mathrm{ref}}\right)$ at this position are evaluated from smoothed phase and modulation curves (see Figure 2). For specified spectrum wavelengths set (usually from 3 to 8 distinctive values for each examined interval) in every recorded fluorescence spectrum, values of phase $(\mathrm{P})$ and modulation $(\mathrm{M})$ are evaluated from corresponding smoothed curves and calculated $\mathrm{P}$ - $\mathrm{P}_{\text {ref }}$ differences, resp. $\mathrm{M} / \mathrm{M}_{\text {ref }}$ ratio are used as an input table for GLOBAL fitting program to calculate single fluorescence lifetime components and their intensity representation.

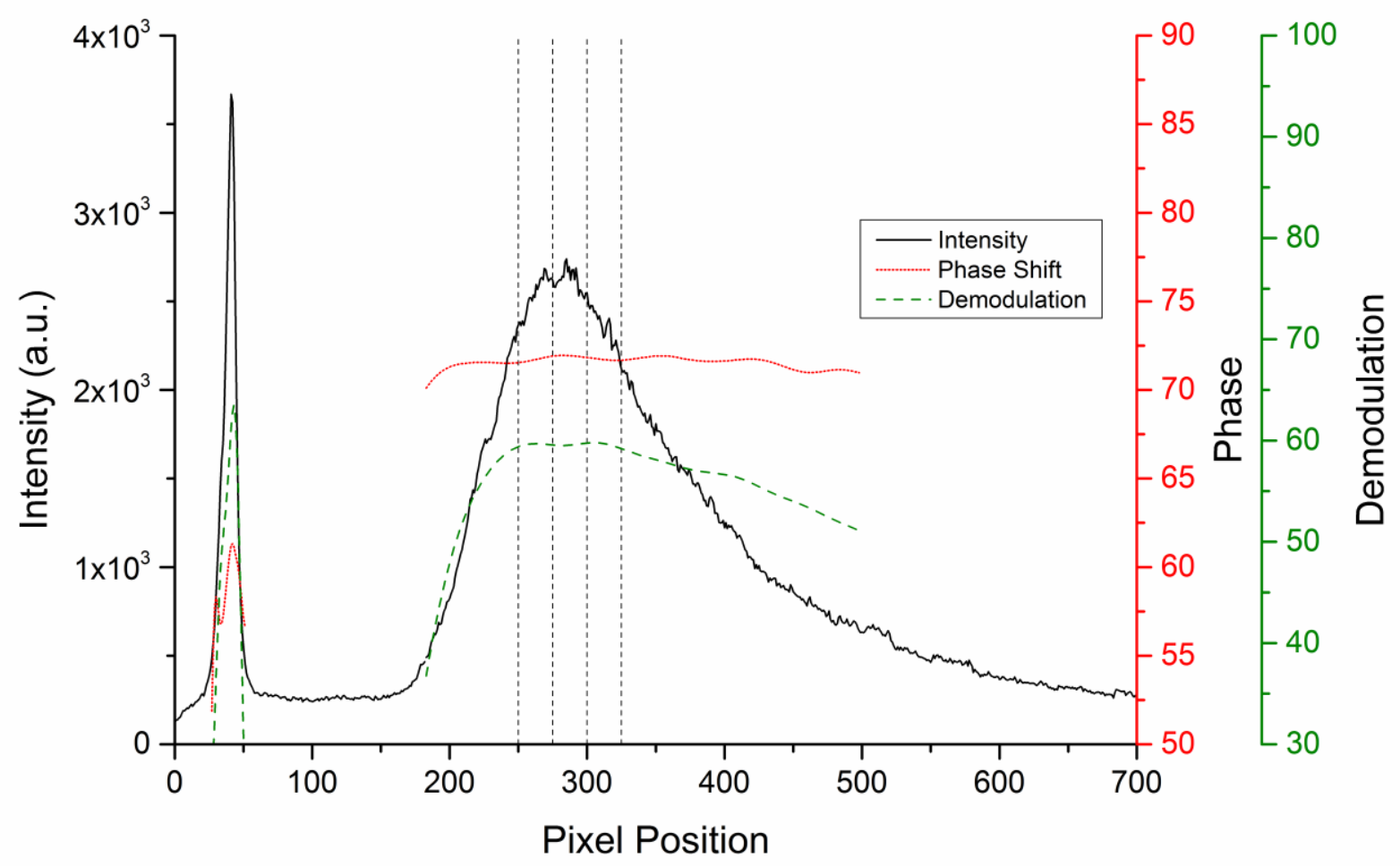


Figure 2 Experimental emission spectrum and corresponding phase and demodulation values (displayed in the part of the elastic scattering and the fluorescence band as well) depending on pixel position of the detector. Vertical dashed lines demonstrate positions at which the reading of phase shift and demodulation was done.

White-light and fluorescence images of the cells were obtained with inverted fluorescence microscope Nikon Eclipse Ti by using an oil-immersion objective 100×/1.3 (Nikon Plan Fluor OFN25 Ph3 DLL). For fluorescence images the excitation (482/35 nm) and emission $(536 / 40 \mathrm{~nm})$ filters were used. This setting enables us to observe intracellular distribution of labeled AS1411 aptamer.

\section{Results and discussion}

Fluorescence micro imaging is typically used as a highly informative technique providing the overall view about the distribution of the labeled molecule of interest throughout the population of cells in the cell line. It indisputably demonstrates the general state of the staining, the presence or absence of the labeled molecule on the surface of the cells and inside them bound to the subcellular structures. Typical fluorescence image from tumoral cells of human glioblastoma line U-87 MG incubated with AS1411 aptamer labeled by ATTO 425 after three hours of incubation is shown in Figure 3. It clearly reveals the aptamer presence throughout the cells with prevalent occurrence in the cytoplasm. Labeled aptamers cross the cellular membrane, spread out in the cell although the cell nuclei rest practically unstained. From the non-homogenous character of the staining we can conclude that uptake process is more likely mediated than simple diffusive. As known from already published results, ${ }^{4}$ tumoral cells possess increased levels of the cytoplasmic and plasma membrane nucleolin in comparison to normal healthy cells. Moreover, nucleolin localized in the plasma membrane is considered to be a very effective shuttle and it enables the aptamer uptake. ${ }^{4}$ It implies an advantage that no additional 'helper system ` is necessary to support the aptamer transport through the cellular membrane. In addition, uptake of labeled AS1411 aptamer into U-87 MG cells doesn't need a long overnight incubation, much shorter time (1-3 hours) is sufficient. 


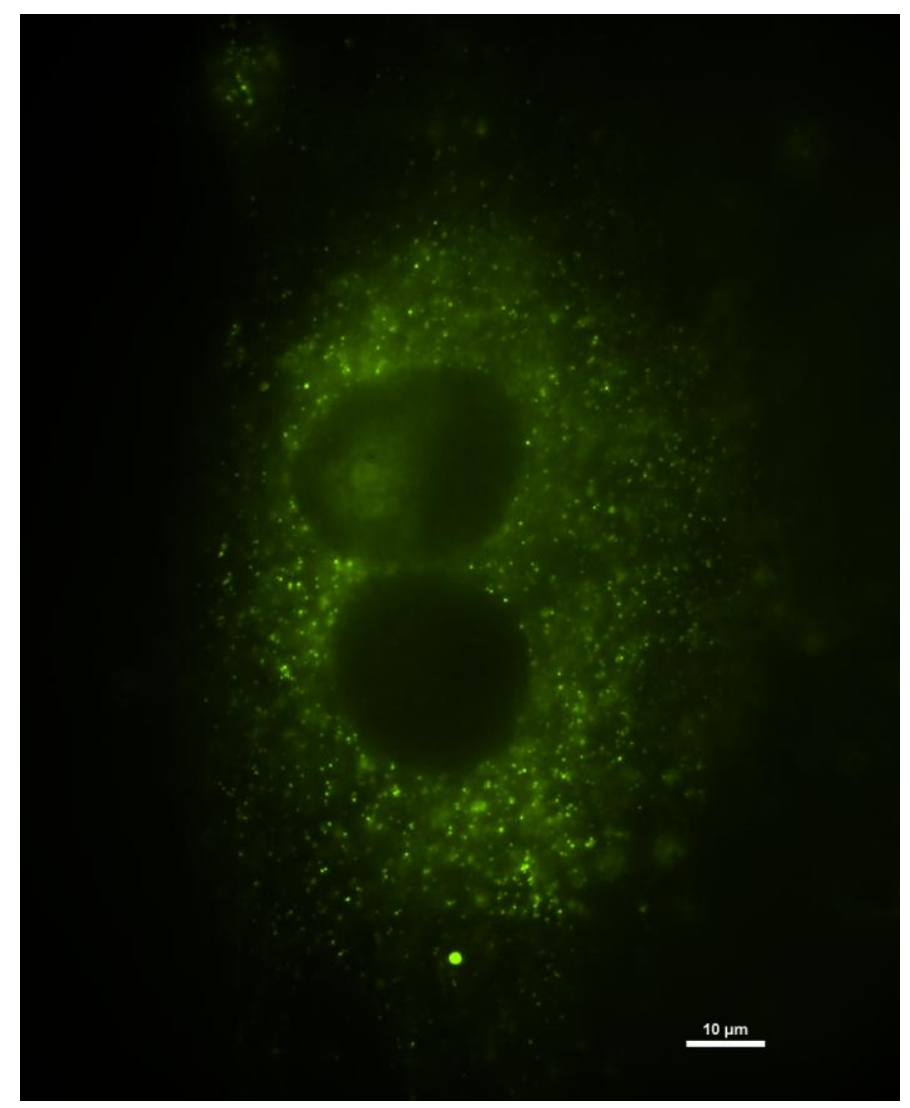

Figure 3 Fluorescence image of the cells from U-87 MG cell line (tumoral human glioblastoma cells) incubated with AS1411 aptamer labeled by ATTO 425. Time of incubation was approximately 3 hours.

Time-resolved microfluorescence data were obtained from a set of U-87 MG cells and from reference aptamer aqueous solutions (or suspensions) as well. The solution measurements are commonly done in order to imitate and simplify in parts the complex cellular environment with the aim to recognize possible molecular targets. Like that, the outcome lead to the easier understanding and interpretation of the data obtained from the cellular environment.

First reference measurement was done on solution of AS1411 aptamer labeled by ATTO 425 in PBS buffer with $\mathrm{K}^{+}$ions needed to form a quadruplex structure. Measured data were processed with our PHR program and subsequently lifetime analysis was performed by GLOBAL fitting procedure to distinguish the lifetime components. Fluorescence emission spectrum obtained from U-87 MG cellular environment with fitted phase shift and demodulation values is shown in Figure 4. Specific lifetime was assessed from the grayish area of the fluorescence band. Single lifetime value of 4.4 ns has been calculated for several 
measurements of aptamer concentration in $\mu \mathrm{M}$ scale. In view of the fact that we are interested in the transport of AS1411 aptamer through the cellular membrane, it is leading us to the consideration of its behavior in the presence of the lipid environment. The model membrane lipid system of liposomes was formed for this study. The mixture of AS1411 aptamer with the liposome suspension (incubated for tens of minutes up to 3 hours) shows single lifetime of 3.7 ns. This value slightly differ from than that one in the aqueous buffer solution and it reveals that environment composed of amphiphilic lipid molecules causes the lifetime shortening.

As for intracellular time-resolved measurements on U-87 MG and Hs68 cell lines, several independent incubations in the presence of the labeled AS1411 aptamer were accomplished. Obtained dataset accumulated from intracellular environment of several different uptaken cells was processed in the same way as in case of the reference measurements in solutions or suspensions. Typical fluorescence emission spectrum observed in U-87 MG cells consists of ATTO 425 labeled aptamer emission band and the lifetime reference elastic scattering peak (see Figure 5). One may observe the same spectral shape in case of Hs68 (recorded spectrum not shown here). Corresponding curves of fitted phase shift and demodulation data clearly indicating single lifetime component can be seen in Figure 5 inset. Calculated fluorescence lifetime value is $5.8 \mathrm{~ns}$ (with the calculation error within +$0.15 \mathrm{~ns})$. As one may note the intracellular lifetime of the labeled AS1411 aptamer in the U$87 \mathrm{MG}$ cells is markedly lengthened when compared to both buffer aptamer solution and liposome suspension in mixture with aptamer values. This result indicates that cellular space in tumoral cell line represents a distinct microenvironment for the aptamer localization. As for Hs68 cell line, calculated fluorescence lifetime shows single $4.3 \mathrm{~ns}$ lifetime component. This lifetime value is quite similar to the labeled aptamer obtained from the solution measurement but it is distinct from that one in tumoral U-87 MG cell line. Thus, a remarkable lifetime change was observed for tumoral cell line in comparison to non-tumoral one in this experiment. 


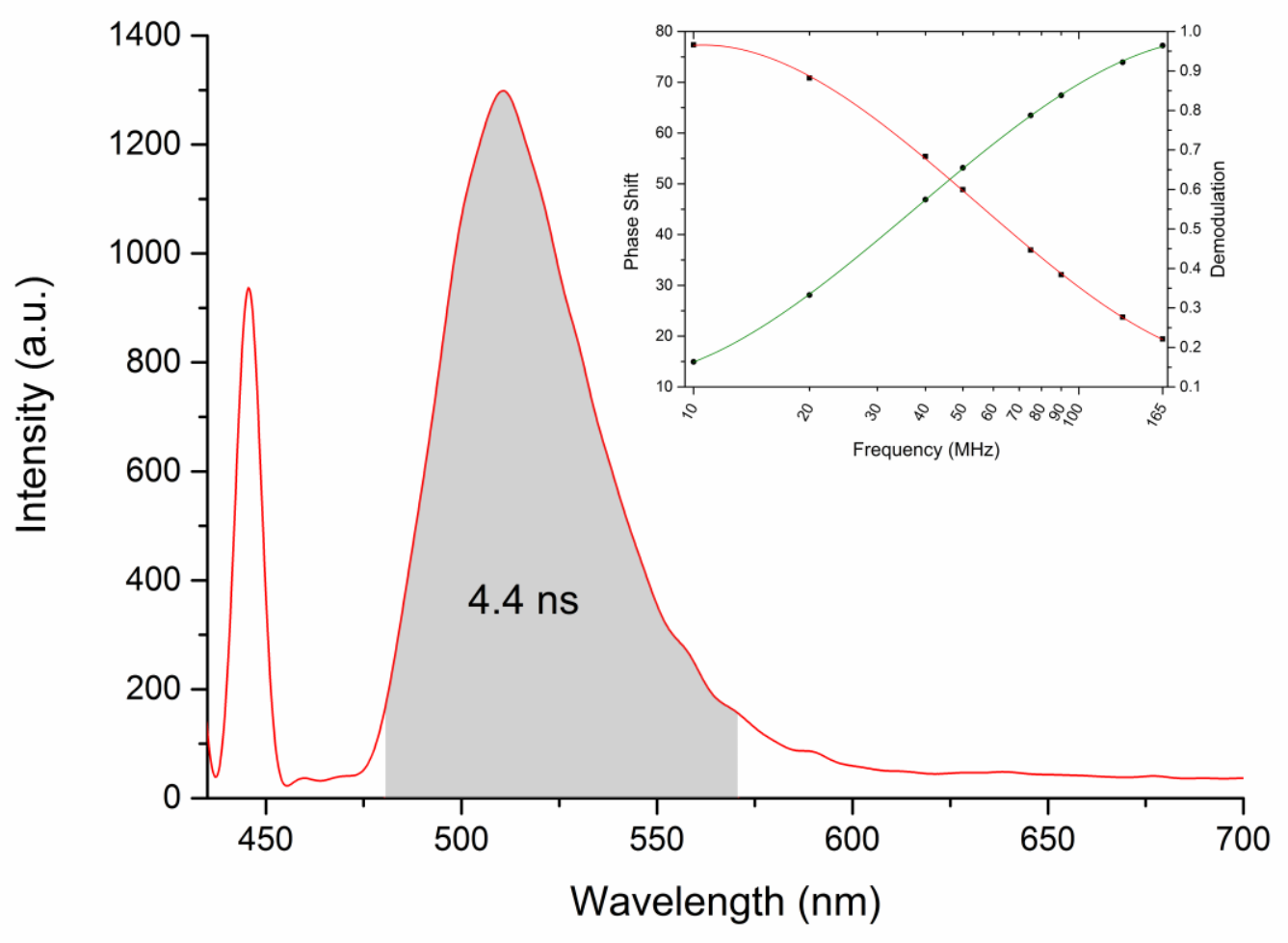

Figure 4 Emission spectrum of AS1411 aptamer labeled by ATTO 425 in PBS buffer solution containing $\mathrm{K}^{+}$ions. Fluorescence lifetime was calculated from grayish part of the fluorescence band. Inset: Fitted phase shift and demodulation data curves indicating single component fluorescence lifetime 


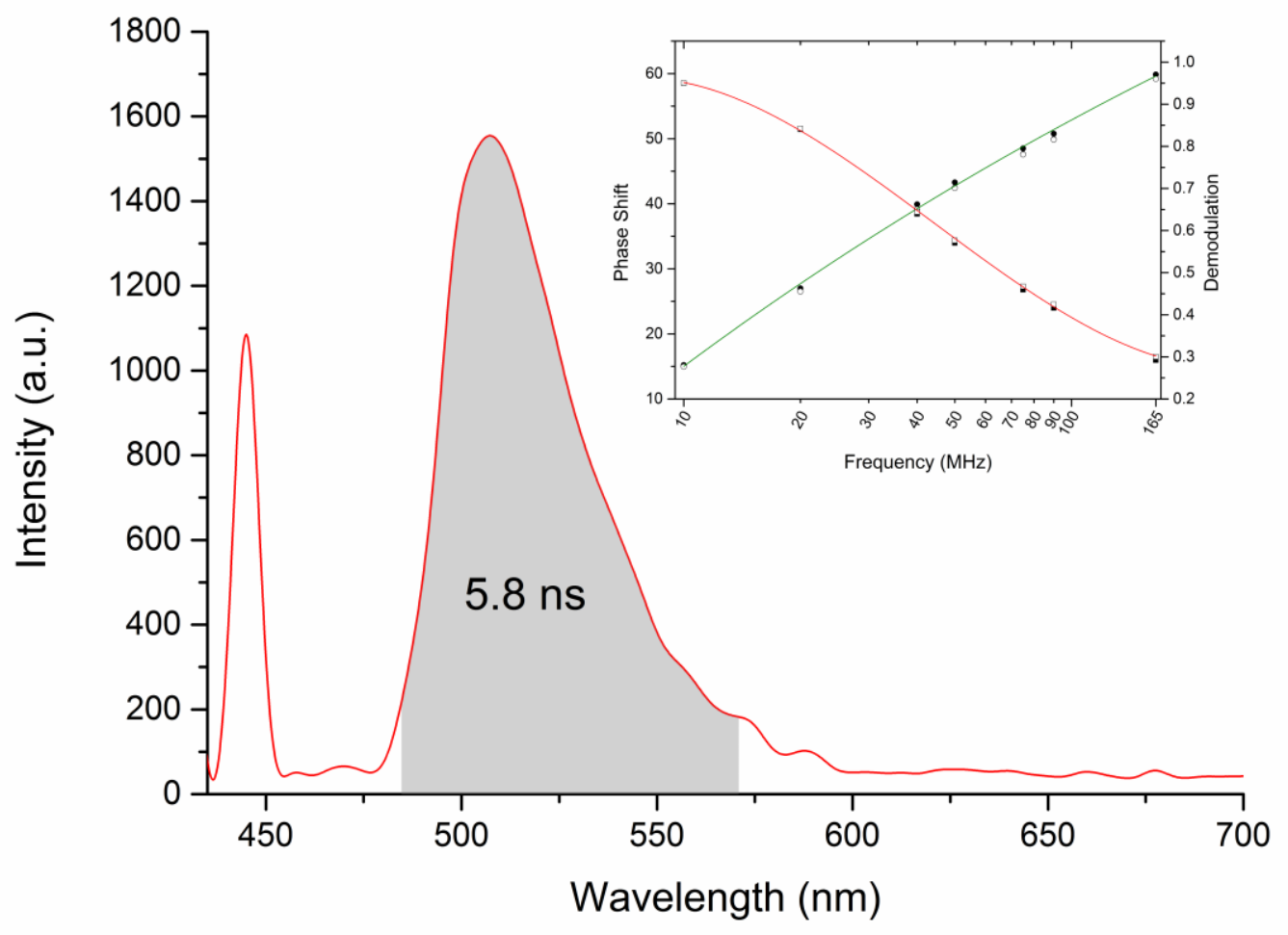

Figure 5 Typical emission spectrum obtained from tumoral U-87 MG cells incubated with AS1411 aptamer labeled by ATTO 425 (wide fluorescence band). Inset: Fitted phase shift and demodulation data curves indicating single component fluorescence lifetime.

\section{Conclusion}

The classic fluorescence micro imaging and time-resolved microspectrofluorimetry were employed to study the AS1411 aptamer transport inside the tumoral and non-tumoral cell lines. The aptamer labeled by ATTO 425 and dissolved in PBS buffer with $\mathrm{K}^{+}$ions gives a single fluorescence lifetime of $4.4 \mathrm{~ns}$. In the lipid environment, its lifetime changes down to $3.7 \mathrm{~ns}$. As for the intracellular measurements it was found the differences in aptamer lifetimes for the tumoral and non-tumoral cell line. Lifetime of U87 MG tumoral cell line is markedly lengthened when compared to that one of the aptamer in solution while the lifetime value of non-tumoral Hs68 cell line does not change considerably.

\section{Acknowledgements}

\section{Reference list}


1. J. Goodchild, "Therapeutic Oligonucleotides", Chap. 1 in Methods in Molecular Biology, Volume 764, Springer Protocols, John Goodchild Ed., pp 1-15, Humana Press, (2011).

2. S. M. Nimjee et al., Aptamers: An emerging class of therapeutics," Annu. Rev. Med. 56, 555-583 (2005).

3. Md. Ashrafuzzaman, "Aptamers as Both Drugs and Drug-Carriers," BioMed Res. Int. 2014, 697923 (2014).

4. P. J. Bates et al., "Discovery and development of the G-rich oligonucleotide AS1411 as a novel treatment for cancer," Exp. Mol. Pathol. 86(3), 151-164 (2009).

5. E. M. Reyes-Reyes et al., "A new paradigm for aptamer therapeutic AS1411 action: uptake by macropinocytosis and its stimulation by a nucleolin-dependent mechanism," Cancer Res. 70(21), 8617-8629 (2010).

6. P. Praus, F. Sureau, “Spectral decomposition of intracellular complex fluorescent signals using multiwavelength phase modulation lifetime determination,” J. Fluor. 10(4), 361-364 P (2000).

7. P. L. T. Tran et al., "Tetramolecular quadruplex stability and assembly" in Quandruplex Nucleic Acids, Topics in Current Chemistry 330, J. B. Chaires and D. Graves Editors, pp. 243-274, Springer-Verlag Berlin Heidelberg \}2012].

8. P. Praus et al., "Advanced microfluorescence methods in monitoring intracellular uptake of “antisense” oligonucleotides," Curr. Org. Chem. 11(6), 515-527 (2007).

9. E. Kocisova et al., "Intracellular uptake of modified oligonucleotide studied by two fluorescence techniques," Biopolymers 74(1-2), 110-114 (2004). 